\title{
¿Es necesario describir los instrumentos empleados en investigaciones empíricas?
}

\author{
Is it necessary to describe the instruments used in empirical research?
}

\author{
Gustavo Alexis Calderón de la Cruz ${ }^{1,2}$, Sergio Alexis Dominguez-Lara ${ }^{1,3}$, \\ Jhonatan S. Navarro Loli ${ }^{1,2}$ \\ Instituto de Investigación de la Escuela Profesional de Psicología de la Universidad de San Martín de Porres, Lima, Perú. \\ 2 Licenciado en Psicología. \\ ${ }^{3}$ Magister en Psicología Clínica y de la Salud.
}

An Fac med. 2016;77(2):175-7 / http://dx.doi.org/10.15381/anales.v77i2.11825

SR. EDITOR:

En la reciente publicación de IzaguirreSotomayor y col. ${ }^{(1)}$, los autores refieren la utilización de una escala para evaluar las actitudes hacia la ética, pero en el apartado 'Métodos', la descripción del instrumento es limitada porque no se reporta información sobre los procesos de obtención de evidencias de validez, estimaciones de la confiabilidad y los baremos de los puntajes. La ausencia de esta información no permite conocer si la escala en mención cumple con los requisitos solicitados por los estándares internacionales de calidad de un test, específicamente en criterios normativos y psicométricos que la constituyen y los efectos de su uso ${ }^{(2)}$.

Asimismo, los autores tampoco dan información sobre antecedentes nacionales o internacionales donde se utilice dicha escala y, por lo tanto, no se conoce réplicas sobre la estructura dimensional, el grado de precisión de los puntajes ${ }^{(3)}$, ni el alcance de las inferencias realizadas a partir de dichos puntajes (4). En consecuencia, los resultados del estudio podrían estar sujetos a cuestionamientos relacionados a la robustez de sus conclusiones.

Por lo tanto, es necesario enfatizar que la divulgación de un manuscrito científico todavía reporta puntos álgidos relacionados a la insuficiencia en la información ${ }^{(5)}$, siendo el apartado de
'Métodos', uno de los puntos sensibles a esta problemática, probablemente debido al grado de facilidad con el que es percibido su desarrollo ${ }^{(6)}$ o quizá por desconocimiento del investigador sobre las condiciones formales en la redacción de un artículo científico ${ }^{(7)}$. En ese sentido, se debe resaltar que la importancia de esta sección, en este tipo de comunicación científica (artículo de investigación), está centrada en contener toda la información necesaria para garantizar la reproducción del estudio por otro investigador ${ }^{(6,8)}$.

Conforme a lo expuesto, es conveniente que los autores esclarezcan y reporten algunos elementos vinculados con la escala, sobre todo en relación a sus propiedades métricas. De lo contrario, el esfuerzo desplegado en el proceso de investigación, elaboración y publicación del manuscrito quizá no tenga el impacto esperado por las omisiones enunciadas anteriormente, poniendo en cuestionamiento las conclusiones del estudio. De igual forma, la incorporación de estos aspectos permitirá que otros investigadores tengan la información suficiente para replicar el estudio y comparar resultados, permitiendo así el avance del conocimiento científico, así como para elevar la calidad del manuscrito ${ }^{(6)}$.

\section{REFERENCIAS BIBLIOGRÁFICAS}

1. Izaguirre-Sotomayor $M H$, Reátegui-Guzmán, LA, Mori-Ramirez H, Robles-Rodriguez FJ, SánchezVilcapuma WJ. An Fac med. 2015;76(4):361-8. doi: http://dx.doi.org/10.15381/anales.v76i4.11405.

2. American Educational Research Association, American Psychological Association \& National Council on Measurement in Education. Standards for educational and psychological testing. Washington, DC: American Educational Research Association. 1999.

3. Meyer JP. Reliability. New York: Oxford University Press; 2010.

4. Furr RM. Scale construction and psychometrics for social and personality psychology. California: SAGE; 2011

5. Catalaya-López F, Hutton B, Page MJ, Vieta E, Tabarés-Seisdedos R, Moher D. Declaración de transparencia: un paso hacia la presentación completa de artículos de investigación. Rev Psiquiatr Salud Ment (Barc.). En prensa. doi: http://dx.doi. org/10.1016/j.rpsm.2015.08.003.

6. Herranz G. Material y método: cosas básicas dichas en letra pequeña. Med Clin (Barc). 1986;86:205-6.

7. Corral de la Calle MA, Encinas de la Iglesia J. Cómo escribir un artículo. Cuestiones formales. Radiología. 2013;55(S1):S17-S27. doi: http:// dx.doi.org/10.1016/j.rx.2013.01.002.

8. Manterola C, Pineda V, Vial M, Grande, L. ¿Cómo presentar los resultados de una investigación científica? II. El manuscrito y el proceso de publicación. Cir Esp. 2007;81(2):70-7. doi: http:// dx.doi.org/10.15381/anales.v76i4.11405.10.1016/ S0009-739X(07)71250-2.

Carta al Editor recibida el 9 de febrero de 2016.

Correspondencia:

Gustavo Alexis Calderón de la Cruz.

Dirección: Av. Tomás Marsano 242 (5to piso), Lima.

Perú- 34

Teléfono: 986612090

Correo electrónico: gcalderond@usmp.pe 
La presente Carta al Editor fue alcanzada al autor corresponsal del artículo 'iEs necesario describir los instrumentos empleados en investigaciones empíricas?', quien escribió la siguiente respuesta, el día 27 de marzo de 2016.

\section{ESTIMADO DR. PACHECO:}

Con referencia a lo manifestado por los investigadores Gustavo Alexis Calderón de la Cruz, Sergio Alexis Dominguez-Lara y Jhonatan S. Navarro Loli en el sentido de la necesidad de describir los instrumentos empleados en toda investigación, estamos de acuerdo con lo manifestado, en virtud de lo cual pedimos disculpas por la lamentable omisión realizada.

Con relación a los antecedentes nacionales e internacionales utilizando el mismo instrumento, no la encontramos. La fiabilidad del instrumento mostrada a través del valor Alfa de Cronbach fue de 0, 845 .

Estadisticas de fiabilidad

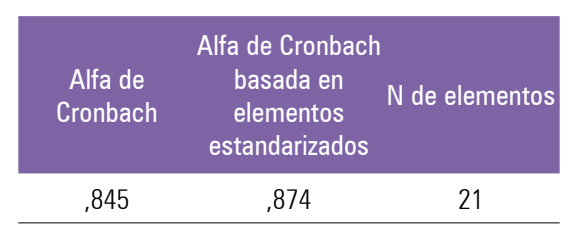

Para interpretar los resultados se construyó la siguiente escala gráfica para el análisis e interpretación de los puntajes obtenido en la escala de Likert por los docentes y estudiantes de Pediatría de la Facultad de Medicina de la Universidad Nacional Mayor de San Marcos, donde los puntajes comprendidos entre 1 y 1,99 correspondieron
Estadísticas de total de elemento

\begin{tabular}{|c|c|c|c|c|}
\hline & $\begin{array}{l}\text { Media de escala si } \\
\text { el elemento se ha } \\
\text { suprimido }\end{array}$ & $\begin{array}{c}\text { Varianza de escala } \\
\text { si el elemento se ha } \\
\text { suprimido }\end{array}$ & $\begin{array}{l}\text { Correlación total } \\
\text { de elementos } \\
\text { corregida }\end{array}$ & $\begin{array}{c}\text { Alfa de Cronbach si } \\
\text { el elemento se ha } \\
\text { suprimido }\end{array}$ \\
\hline VAR 1 & 85,0500 & 62,155 & ,490 & 836 \\
\hline VAR 2 & 85,0500 & 61,945 &, 508 & 835 \\
\hline VAR 3 & 84,7500 & 62,408 & ,636 & 832 \\
\hline VAR 4 & 84,8000 & 64,589 & ,485 & 838 \\
\hline VAR 5 & 85,2000 & 56,379 & ,635 & 827 \\
\hline VAR 6 & 84,8000 & 66,484 & ,252 & 844 \\
\hline VAR 7 & 85,9500 & 61,629 & ,332 &, 845 \\
\hline VAR 8 & 85,2000 & 63,642 & 301 & 844 \\
\hline VAR 9 & 84,9000 & 62,305 & 655 & 832 \\
\hline VAR 10 & 85,4000 & 56,042 & ,727 & 822 \\
\hline VAR 11 & 85,0000 & 61,474 &, 418 & 839 \\
\hline VAR 12 & 84,8500 & 61,503 &, 569 & 833 \\
\hline VAR 13 & 85,0500 & 68,155 &,- 009 & 858 \\
\hline VAR 14 & 84,9500 & 63,208 & 695 & 833 \\
\hline VAR 15 & 84,9000 & 62,516 & 632 & 832 \\
\hline VAR 16 & 85,5000 & 68,053 &,- 013 & 860 \\
\hline VAR 17 & 85,0500 & 63,945 & ,441 & .838 \\
\hline VAR 18 & 84,9000 & 64,832 & 380 & 840 \\
\hline VAR 19 & 84,8500 & 62,555 & ,748 & 831 \\
\hline VAR 20 & 84,8500 & 62,661 & ,608 & 833 \\
\hline VAR 21 & 85,0000 & 65,158 & , 173 & 851 \\
\hline
\end{tabular}

a una actitud muy desfavorable, los incluidos entre 2 y 2,99 a una actitud desfavorable, aquellos entre 3 y 3,99 correspondieron a una actitud indiferente, los puntajes de 4 a 4,99 a una actitud favorable y el puntaje 5 correspondió a una actitud muy favorable. Figura 1.

Finalmente con respecto a los baremos de los puntajes conseguidos por los docentes en general y los obtenidos según sexo (F-M), condición de trabajo $(\mathrm{N}-\mathrm{C})$, edad $(20-35 ; 36-50$ y 51 a $+)$ y años de experiencia (1-10; 11-20; 21-30 y $31 \mathrm{a}+$ ), así como los alcanzados por los estudiantes en general y

\begin{tabular}{|l|c|c|c|c|}
\hline \multicolumn{1}{|c|}{1 a 1,99} & 2 a 2,99 & 3 a 3,99 & 4 a 4,99 & 5 \\
\hline Muy desfavorable & Desfavorable & Indiferente & Favorable & Muy favorable \\
\hline ACTITUD & \multicolumn{3}{|l}{} & \\
\hline Negativa & & & Positiva \\
\hline
\end{tabular}

Figura 1. Escala gráfica para el análisis e interpretación de los puntajes obtenidos en la escala de Likert por los docentes y estudiantes de pediatria de la facultad de medicina UNMSM 2014.
Comunalidades

\begin{tabular}{ccc} 
& Inicial & Extracción \\
VAR 1 & 1,000 &, 958 \\
VAR 2 & 1,000 &, 894 \\
VAR 3 & 1,000 &, 932 \\
VAR 4 & 1,000 &, 825 \\
VAR 5 & 1,000 &, 801 \\
VAR 6 & 1,000 &, 878 \\
VAR 7 & 1,000 &, 830 \\
VAR 8 & 1,000 &, 904 \\
VAR 9 & 1,000 &, 858 \\
VAR 10 & 1,000 &, 922 \\
VAR 11 & 1,000 &, 691 \\
VAR 12 & 1,000 &, 800 \\
VAR 13 & 1,000 &, 792 \\
VAR 14 & 1,000 &, 833 \\
VAR 15 & 1,000 &, 931 \\
VAR 16 & 1,000 &, 878 \\
VAR 17 & 1,000 &, 842 \\
VAR 18 & 1,000 &, 780 \\
VAR 19 & 1,000 &, 865 \\
VAR 20 & 1,000 &, 629 \\
VAR 21 & 1,000 &, 781 \\
\hline
\end{tabular}

Método de extracción: análisis de componentes principales. 
los obtenidos según sexo (F- M), edad (20-29; 30-40 y $41 \mathrm{a}+)$ y años de experiencia $(1-6 ; 7-8 ; 09-10$ y 11 a + ), les remitimos solamente los baremos de los puntajes obtenidos por los docentes y los estudiantes en general, en mérito al espacio que ocuparía.
Tenga por seguro estimado Dr. Pacheco que esta lamentable omisión no volverá a ocurrir.

Atentamente,

Prof. Manuel Izaguirre S.

Correo electrónico: manuel.izaguirre@gmail.com

Baremo de los puntajes obtenidos por los docentes

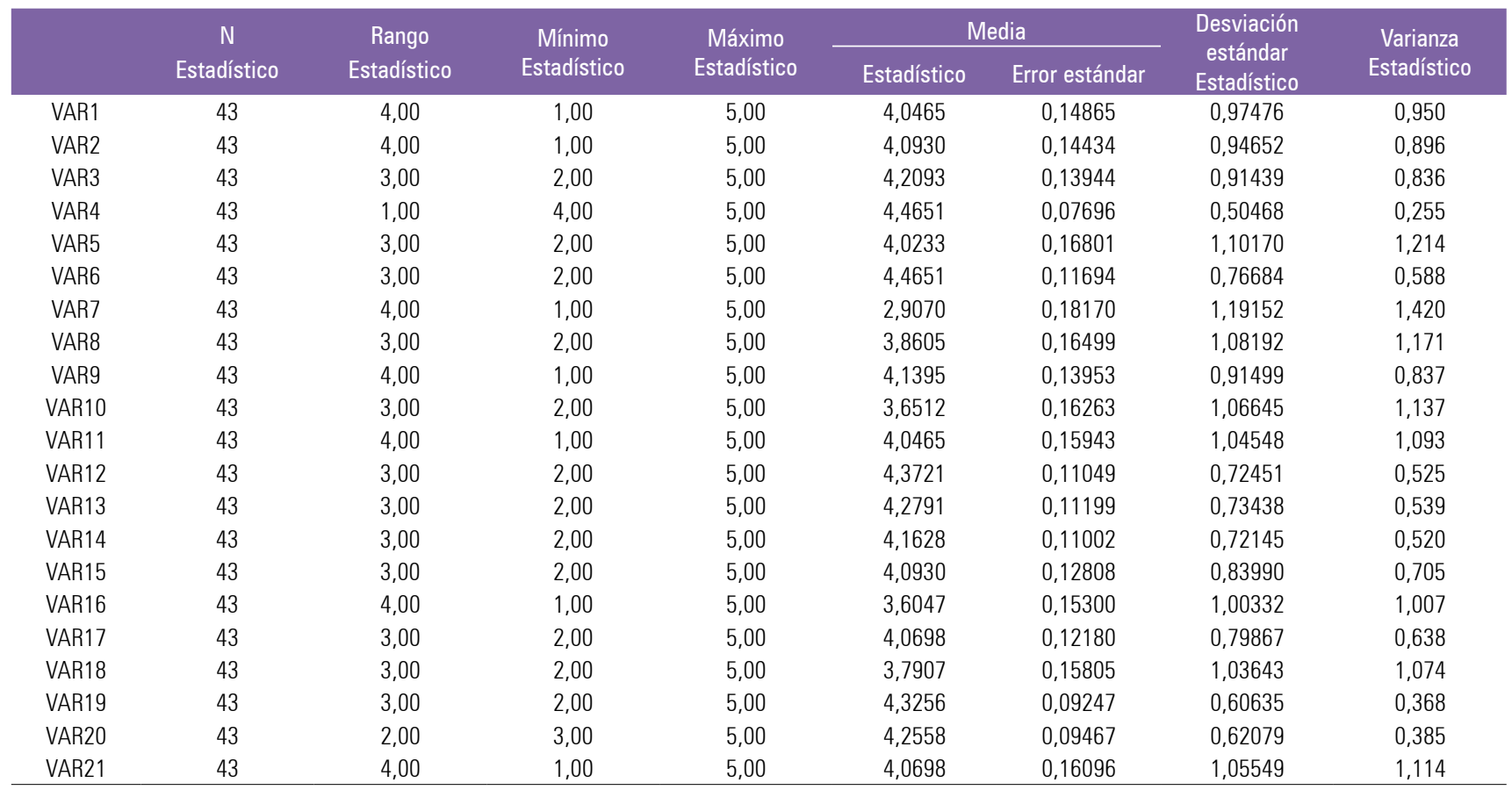

Baremo de los puntajes obtenidos por los estudiantes

\begin{tabular}{|c|c|c|c|c|c|c|c|c|}
\hline & \multirow{2}{*}{$\begin{array}{c}\mathrm{N} \\
\text { Estadístico }\end{array}$} & \multirow{2}{*}{$\begin{array}{c}\text { Rango } \\
\text { Estadístico }\end{array}$} & \multirow{2}{*}{$\begin{array}{l}\text { Mínimo } \\
\text { Estadístico }\end{array}$} & \multirow{2}{*}{$\begin{array}{l}\text { Máximo } \\
\text { Estadístico }\end{array}$} & \multicolumn{2}{|c|}{ Media } & \multirow{2}{*}{$\begin{array}{l}\text { Desviación } \\
\text { estándar } \\
\text { Estadístico }\end{array}$} & \multirow{2}{*}{$\begin{array}{c}\text { Varianza } \\
\text { Estadístico }\end{array}$} \\
\hline & & & & & Estadístico & Error estándar & & \\
\hline VAR1 & 96 & 2,00 & 3,00 & 5,00 & 4,3542 & 0,05731 & 0,56156 & 0,315 \\
\hline VAR2 & 96 & 3,00 & 2,00 & 5,00 & 4,3333 & 0,06893 & 0,67538 & 0,456 \\
\hline VAR3 & 96 & 4,00 & 1,00 & 5,00 & 4,1146 & 0,07957 & 0,77961 & 0,608 \\
\hline VAR4 & 96 & 3,00 & 2,00 & 5,00 & 4,1979 & 0,06886 & 0,67465 & 0,455 \\
\hline VAR5 & 96 & 3,00 & 2,00 & 5,00 & 3,7708 & 0,09652 & 0,94567 & 0,894 \\
\hline VAR6 & 96 & 3,00 & 2,00 & 5,00 & 4,1458 & 0,07099 & 0,69554 & 0,484 \\
\hline VAR7 & 96 & 4,00 & 1,00 & 5,00 & 3,0000 & 0,10576 & 1,03619 & 1,074 \\
\hline VAR8 & 96 & 4,00 & 1,00 & 5,00 & 3,2292 & 0,10830 & 1,06107 & 1,126 \\
\hline VAR9 & 95 & 4,00 & 1,00 & 5,00 & 3,6947 & 0,10158 & 0,99010 & 0,980 \\
\hline VAR10 & 96 & 4,00 & 1,00 & 5,00 & 3,9479 & 0,07310 & 0,71627 & 0,513 \\
\hline VAR11 & 96 & 4,00 & 1,00 & 5,00 & 4,1354 & 0,08961 & 0,87803 & 0,771 \\
\hline VAR12 & 96 & 3,00 & 2,00 & 5,00 & 4,2500 & 0,06623 & 0,64889 & 0,421 \\
\hline VAR13 & 96 & 3,00 & 2,00 & 5,00 & 4,1250 & 0,07292 & 0,71451 & 0,511 \\
\hline VAR14 & 96 & 3,00 & 2,00 & 5,00 & 4,0833 & 0,05861 & 0,57430 & 0,330 \\
\hline VAR15 & 96 & 4,00 & 1,00 & 5,00 & 3,8438 & 0,07598 & 0,74450 & 0,554 \\
\hline VAR16 & 96 & 4,00 & 1,00 & 5,00 & 3,7396 & 0,08792 & 0,86139 & 0,742 \\
\hline VAR17 & 96 & 4,00 & 1,00 & 5,00 & 4,0521 & 0,08161 & 0,79960 & 0,639 \\
\hline VAR18 & 96 & 4,00 & 1,00 & 5,00 & 3,8229 & 0,09247 & 0,90606 & 0,821 \\
\hline VAR19 & 96 & 4,00 & 1,00 & 5,00 & 4,0625 & 0,08220 & 0,80541 & 0,649 \\
\hline VAR20 & 96 & 3,00 & 2,00 & 5,00 & 4,0417 & 0,06933 & 0,67927 & 0,461 \\
\hline VAR21 & 96 & 3,00 & 2,00 & 5,00 & 3,9062 & 0,08122 & 0,79575 & 0,633 \\
\hline
\end{tabular}

